\title{
Identification and differential expression patterns of porcine OCT4 variants
}

\author{
Jae Yeon Hwang ${ }^{1}$, Jong-Nam Oh ${ }^{1}$, Dong-Kyung Lee ${ }^{1}$, Kwang-Hwan Choi ${ }^{1}$, Chi-Hun Park ${ }^{2}$ \\ and Chang-Kyu Lee ${ }^{1,2}$ \\ ${ }^{1}$ Department of Agricultural Biotechnology, Animal Biotechnology Major, Research Institute of Agriculture and \\ Life Sciences, Seoul National University, Seoul 151-921, Korea and ${ }^{2}$ Institute of Green Bio Science and Technology, \\ Seoul National University, Kangwon-do 232-916, Korea \\ Correspondence should be addressed to C-K Lee; Email: leeck@snu.ac.kr
}

\begin{abstract}
OCT4 encoded by POU5F1 has a crucial role of maintaining pluripotency in embryonic stem cells during early embryonic development and several $O C T 4$ variants have been identified in mouse and human studies. The objective of this study was to identify different variants of $O C T 4$ and analyze their expression patterns in preimplantation porcine embryos and various tissues. In this study, we showed that POU5F1 transcribes its three variants, namely OCT4A, OCT4B, and OCT4B1. The OCT4B transcript consists of exons identical to the major form of the $O C T 4$ variant, $O C T 4 A$, with a differential $\mathrm{N}$-terminal domain-coding exon. The structure of $O C T 4 B 1 \mathrm{mRNA}$ was the same as that of $O C T 4 B$ mRNA, but harbored a cryptic exon. Based on these findings, the transcription levels were investigated and found that $O C T 4 B$ and $O C T 4 B 1$ made up $\sim 20 \%$ among the variants in the embryonic stage and this indicates that $O C T 4 A$ mRNA is dominantly expressed during preimplantation embryo development. In addition, OCT4B mRNA was detected in all tissues examined, while $O C T 4 A$ and $O C T 4 B 1$ were detected only in testis but not in other tissues examined. OCT4B1 showed inversely correlated expression with SOX2 and NANOG expression. OCT4A protein was specifically localized to the nuclei, whereas OCT4B was mainly localized to the cytoplasm of the porcine embryos at the blastocyst stage. The findings of this study reveal that the porcine OCT4 gene can potentially encode three variants $(O C T 4 A, O C T 4 B$, and $O C T 4 B 1)$, and they are differentially expressed and would have roles dissimilar between each other in preimplantation embryos and various adult tissues.

Reproduction (2015) 149 55-66
\end{abstract}

\section{Introduction}

The transcription factor OCT4 (also named POU5F1) belongs to a protein family encoding the octamer-binding Pit-Oct-Unc (POU) domain (Scholer et al. 1990, Takeda et al. 1992). The Oct4 gene has been shown to be expressed in the early developmental embryonic stage, embryonic carcinoma (EC) cell, embryonic stem (ES) cell, and germ cell lineage (Okamoto et al. 1990, Rosner et al. 1990, Pesce et al. 1998). Oct4A, which is the most well known form among the Oct4 variants as it is a representative marker for pluripotency, has been suggested to be a gatekeeper for maintaining pluripotency in ES cells and is essential for inner cell mass (ICM) formation during embryo development in mice (Nichols et al. 1998, Niwa et al. 2000). Deletion of Oct4 induces failure of pluripotent ICM formation in the blastocyst (Nichols et al. 1998) and down- or up-regulation of Oct4 makes ES cells differentiate into trophectodermal cells or mesodermal cells respectively (Niwa et al. 2000).
The OCT4 gene has also been suggested as one of the essential transcription factors for reprogramming somatic cells and inducing those to have pluripotency (Takahashi \& Yamanaka 2006, Takahashi et al. 2007). These studies have clearly demonstrated the importance of the OCT4 gene, especially the A form, in embryo development and ES cell pluripotency.

Human and mouse OCT4 variants, OCT $4 B$ and OCT4B1, have been identified (Takeda et al. 1992, Atlasi et al. 2008, Mizuno \& Kosaka 2008) and their expressions have been examined in preimplantation embryos, ES cells, EC cells, somatic cells, and various cancer cells (Cauffman et al. 2006, Lee et al. 2006a, Atlasi et al. 2008, Mizuno \& Kosaka 2008, Guo et al. 2012). The OCT4B transcript shares identical exons coding the $\mathrm{POU}$ DNA-binding and C-terminal transactivation domains (CTDs) of OCT4A, but the variants have different exon-coding $\mathrm{N}$-terminal transactivation domains (NTDs) (Takeda et al. 1992, Mizuno \& Kosaka 2008). Moreover, OCT4B1, which is composed of the same 
exons with $O C T 4 B$, contains an additional cryptic exon, which is an intron region in OCT $4 A$ and OCT $4 B$ transcripts (Atlasi et al. 2008, Wang \& Dai 2010).

The functions of the variants of OCT4 are still poorly understood. The relationship between pluripotency and the $B$ and $B 1$ forms of OCT4 has been reported in only a few studies (Lee et al. 2006a, Atlasi et al. 2008, Guo et al. 2012, Mirzaei et al. 2014). These studies demonstrated that OCT4B does not appear to be related to pluripotency (Lee et al. 2006a, Guo et al. 2012). However, one study originally defining OCT4B1 in human suggested that the $O C T 4 B 1$ variant could have a relationship with pluripotency by comparing expression levels among EC, ES, and somatic cells. These studies suggested that the novel OCT4 variants would have distinctive functions compared with OCT4A. However, little information exists so far on the OCT4 expression of the different variants in pig species. Therefore, this study established undefined variants, OCT4B and OCT4B1, and determined the expression patterns of them in various somatic tissues and embryonic stage in pig.

\section{Materials and methods \\ Ethics statement}

The experimental use of pigs was approved by the Institute of Laboratory Animal Resources, Seoul National University.

\section{BLAST search}

The identification of the human OCT4 and porcine OCT4A mRNA homolog region in the porcine genome was performed following our previous report (Hwang et al. 2013a). Briefly, the sequence of the entire porcine genome (Sus scrofa genome, version 10.2) was compared with the human OCT4 sequence (GenBank accession no.: 5460) using BLAST. The regions with BLAST hit scores of over 100 in the porcine OCT4 genomic region were considered to be sequence homolog regions. The first exon of the porcine OCT4 sequence (GenBank accession no.: NM_001113060.1) was compared with the whole porcine genome and the hit region on the other chromosome was considered to be the porcine OCT4A homolog region. The identified genomic sequences were applied to a porcine OCT4A-specific primer design.

\section{Chemicals}

Unless otherwise stated, all chemicals were purchased from Sigma-Aldrich Corp.

\section{Sample preparation}

Adult tissue

Six adult tissues, liver, lung, kidney, heart, spleen, and testis obtained from a purchased crossbred (Landrace $\times$ Large White $\times$ Duroc $)$ piglet $(d=1, w t=1.5 \mathrm{~kg}$, Jacob farm,
Eumseong, Korea) were ground, frozen in liquid nitrogen, and preserved at $-70{ }^{\circ} \mathrm{C}$ until used in the experiment.

\section{IVF embryo}

IVF embryos were obtained following the method described in our previous study (Hwang et al. 2013b). Briefly, the prepubertal gilt ovaries donated by the Sooam Biotech Research Institute (Seoul, Korea) were used in this experiment. The follicular fluid and cumulus-oocyte complexes (COCs) were aspirated using an 18-gauge needle and pooled to obtain sediments. Sediments were washed with TL-Hepes-PVA medium (Funahashi et al. 1997) and the oocytes with compact cumulus cells and granulated cytoplasm were selected for in vitro maturation. The washed COCs were cultured in a tissue culture medium (TCM-199; Life Technologies) containing $10 \mathrm{ng} / \mathrm{ml}$ epidermal growth factor (EGF), $1 \mu \mathrm{g} / \mathrm{ml}$ insulin, and $10 \%$ PFF for $44 \mathrm{~h}$ at $39{ }^{\circ} \mathrm{C}$ in $5 \% \mathrm{CO}_{2}$ and $100 \%$ humidity conditions. The COCs were treated with hormones, $4 \mathrm{IU} / \mathrm{ml}$ equine chorionic gonadotropin (eCG) and human chorionic gonadotropin (hCG) (Intervet, Cambridge, UK), only for the first $22 \mathrm{~h}$, and then the COCs were maturated in hormone-free conditions. After $44 \mathrm{~h}$ of maturation, cumulus cells were detached from the oocytes using $0.1 \%(\mathrm{w} / \mathrm{v})$ hyaluronidase with gentle pipetting. After removing the cumulus cell, $\sim 20$ mature oocytes were placed into 40- $\mu$ l microdrops of the modified Tris-buffered medium (mTBM; Abeydeera \& Day 1997) and covered with $39^{\circ} \mathrm{C}$ mineral oil. Fresh semen in Duroc (provided by the DARBI A.I. Center in Jochiwon, Korea) was washed by centrifugation with $0.1 \%(\mathrm{w} / \mathrm{v})$ BSA-supplemented PBS and introduced by microdrops for a final concentration of $1 \times 10^{5} \mathrm{cells} / \mathrm{ml}$. The semen and oocytes were co-incubated for $6 \mathrm{~h}$ and the attached sperm cells were removed from the oocyte by repetitive pipetting. Spermremoved fertilized embryos were transferred to the culture medium, porcine zygote medium 3 (PZM-3; Yoshioka et al. 2002). The cleaved and non-cleaved embryos were divided after $48 \mathrm{~h}$ of culture. Embryos of each stage were pooled after zona pellucida were removed by acid Tyrode's solution.

\section{RNA extraction}

\section{Oocyte and preimplantation embryo}

Pooled oocytes $(n=40)$, each stage of pooled IVF embryos (2-cell, $n=20$; 4-cell, $n=20$; 8 -cell, $n=20$; morula, $n=10$; and blastocyst, $n=5$ ), and a single blastocyst were assessed using the Dynabeads mRNA DIRECT Kit (Invitrogen) according to the manufacturer's instructions. The zona pellucida was removed with acidified Tyrode's solution before mRNA extraction.

\section{Somatic tissue}

RNA extraction from adult tissue samples was carried out with TRIzol reagent (Invitrogen) according to the manufacturer's instructions. The RNA extracted from tissues was treated with Turbo DNase I (Applied Biosystems) according to the manufacturer's instructions. 


\section{RT}

DNase-treated RNA from adult tissue $(1 \mu \mathrm{g})$, pooled oocytes and embryos, and a single blastocyst was reverse transcribed using a High Capacity RNA-to-cDNA Kit (Applied Biosystems) in $20 \mu \mathrm{l}$ of the final volume according to the manufacturer's instructions. RNA samples not treated with reverse transcriptase were used as negative controls for genomic DNA contamination.

\section{Genomic DNA extraction}

Genomic DNA from ground liver ( $5 \mathrm{mg}$ ) was extracted using a G-spin Genomic DNA Extraction Kit (iNtRON Bio Technology, Seongnam, Korea) according to the manufacturer's instructions. The gDNA thus obtained was assessed using the porcine OCT4A-specific primer test.

\section{PCR}

Extracted gDNA and reverse-transcribed cDNA were subjected to PCR with the listed primers (Supplementary Table 1, see section on supplementary data given at the end of this article). The amplification was carried out using a $2 \times$ PCR master mix solution (i-MAX II; iNtRON Bio Technology) containing $1 \mu \mathrm{l}$ of cDNA or $100 \mathrm{ng}$ of gDNA samples and $0.5 \mu \mathrm{M}$ concentrations of each primer set in $10-\mu \mathrm{l}$ final reaction volumes with the conditions described in Supplementary Table 2. Amplicons were loaded on the $1.5 \%$ agarose gels stained using ethidium bromide (EtBr).

\section{$5^{\prime}$ - and $3^{\prime}$-RACE PCR}

To identify the transcription starting site (TSS) and the last sequence of porcine OCT4 variant transcripts, RACE PCR was carried out using the $5^{\prime}$ - and $3^{\prime}$-RACE Core Set (Takara Bio, Otsu, Japan) according to the manufacturer's instructions. RNA from pooled IVF blastocysts $(n=20)$ was assessed by both types of RACE PCR, as follows.

\section{5'-RACE PCR}

RNA was reverse transcribed with the primer, 5'-AAGGCAGAGGACATG-3', which phosphorylates the first sequence for ligation. Prepared circular forms of the cDNA sample were subjected to PCR with $1 \mu \mathrm{M}$ of each primer, forward: 5'-GGAAAGGGGGTGGGG-3' and reverse: 5'-TCTGTGGGGGTATCTGTAGGC-3'. Nested PCR was performed with $1 \mu \mathrm{l}$ of PCR product and $1 \mu \mathrm{M}$ of each primer, forward: 5'-GTGGGGTGGGTAAGTGGTA-3' and reverse: 5'-TTATTTGAAGGTCATTTACTCCAGA-3'. Detailed PCR conditions are described in the Supplementary materials and methods section, see section on supplementary data given at the end of this article.

\section{$3^{\prime}-$ RACE PCR}

RNA was reverse transcribed using an adopter oligomer supplied by the kit. PCR and nested PCR were carried out using the forward primer pair, 5'-AGAACCGAGTGAGAGGCAAC-3' and 5'-GCCCAAAGCCCACTCTG-3' respectively. The suggested reverse primer from the 3'-RACE Core Set (Takara Bio) was used for both amplifications. Detailed PCR conditions are described in the Supplementary materials and methods.

\section{Quantitative RT-PCR}

Quantitative PCR was performed using the DyNAmo HS SYBR Green qPCR Kit (Thermo Scientific, Rockford, IL, USA) and the ABI 7300 Real-Time PCR system (Applied Biosystems). For amplification, $0.1 \mu \mathrm{M}$ of each primer listed in Supplementary Table 3, see section on supplementary data given at the end of this article and $0.5 \mu \mathrm{l}$ of cDNA samples from pooled embryos or a single blastocyst were added to the $10-\mu$ l reaction mixtures. The reactions were carried out under the following conditions: 1 cycle of $95{ }^{\circ} \mathrm{C}$ for $10 \mathrm{~min}$ and 40 cycles of $95{ }^{\circ} \mathrm{C}$ for $15 \mathrm{~s}$, at annealing temperatures listed in Supplementary Table 3 for $60 \mathrm{~s}$. The dissociation curve was analyzed to confirm the specificity of the PCR products and the amplified products were loaded on gels. For relative comparison of quantity, ACTB was used as a control gene.

\section{Absolute quantification of the transcripts}

To determine the transcript copy number at each stage of the embryo, standard curves of the target genes were developed. The OCT4 variant amplicons were purified using the MEGAquickspin Total Fragment DNA Purification Kit (iNtRON Bio Technology) and the concentration of each purified amplicon was measured (Supplementary Table 4, see section on supplementary data given at the end of this article). Each purified PCR product was serially diluted by tenfold. Diluted aliquots were assessed by quantitative PCR and the $C t$ values of the five-step serial dilution $\left(10^{-5}-10^{-9}\right.$ dilution) were plotted to show the log of dilution rate of each PCR product with $R^{2}>0.997$ (Supplementary Figure 1 and Supplementary Table 4). As all amplification reactions were performed using $0.5 \mu \mathrm{l}$ of sample, the copy numbers in $0.5 \mu \mathrm{l}$ of PCR product were calculated and the number of mRNA copies in $0.5 \mu$ of sample was calculated using a developed plot with the following equation:

Copy number of $0.5 \mu \mathrm{l}$ sample cDNA

$$
=\text { Copy number of } 0.5 \mu \mathrm{l} \mathrm{PCR} \text { product } \times 10^{\frac{\left(C_{t}-Y_{\text {intercept }}\right.}{\text { Slope }}} \text {. }
$$

After calculating the copy number of a $0.5 \mu$ l sample of cDNA, the copy number of each variant in an oocyte/embryo was calculated as follows:

\section{One oocyte or embryo copy number

$$
=\frac{\text { Copy number of } 0.5 \mu \mathrm{l} \text { sample cDNA } \times 40}{\text { Number of cells or embryos in each pooled sample }} \text {. }
$$

The slope of the developed linear repression plot was in the range of -3.6586 to -3.4727 and was applied to calculate the amplification efficiency $(E)$ with the following equation:

Amplification efficiency $(E)=10^{\left(\frac{-1}{\text { slope }}\right)}$. 
The amplification efficiency ranged from 1.876 to 1.940 (Supplementary Table 4).

\section{Cloning and sequencing}

Gel-loaded PCR products were purified using the MEGAquickspin Total Fragment DNA Purification Kit (iNtRON Bio Technology) to confirm the sequences. Purified DNA fragments were cloned into a PLUG-prime TA-cloning vector kit (iNtRON Bio Technology) and transformed into Escherichia coli cells (DH5 $\alpha$, Novagen, Madison, WI, USA). Plasmid was prepared using commercial spin columns (DNA-spin Plasmid DNA Purification Kit; iNtRON Bio Technology). Purified plasmid samples were sequenced using an ABI PRISM 3730 DNA Analyzer (Applied Biosystems).

\section{Western blot}

The ground pig tissues were lysed with RIPA buffer (SigmaAldrich) to extract total protein. Extracted protein of $50 \mu \mathrm{g}$ was subjected to SDS-PAGE and transferred onto a nitrocellulose transfer membrane (Schleicher \& Schuell Bioscience, Dassel, Germany). The membrane was blocked with $5 \%(w / v)$ skim milk for $1 \mathrm{~h}$ at room temperature. Three NTD-targeting anti-OCT4 primary antibodies, mouse-anti-OCT4 (1:200, SC-5279, SantaCruz); goat-anti-OCT4 (1:200, SC-8628, SantaCruz); rabbit-anti-OCT4 (1:200, SC-9081, SantaCruz), and CTD-targeting goat-anti-OCT4 (1:200, SC-8629, SantaCruz) were used after membrane blocking. Rabbit-anti-ACTIN (1:500, A2066, Sigma-Aldrich) and rabbit-anti-GAPDH (1:500, 5174, Cell Signaling Technology, Boston, MA, USA) were used as internal controls. The membrane was treated with primary antibodies overnight at $4{ }^{\circ} \mathrm{C}$. After incubation, the membrane was washed three times with TBS-T and incubated with goat-anti-mouse IgG-HRP (1:1000, sc-2005, SantaCruz) for SC-5279, donkey-anti-goat IgG-HRP (1:1000, sc-2020, SantaCruz) for SC-8628 and 8629, and goat-anti-rabbit IgG-HRP (1:1000, SC-2004, SantaCruz) for SC-9081, GAPDH, and ACTIN for $1 \mathrm{~h}$ at room temperature. The membrane was then washed three times after secondary antibody treatment and the protein expression was detected by ECL technology (Amersham Bioscience) and visualized by ChemiDoc (ChemiDoc XRS + System, Bio-Rad).

\section{Immunocytochemistry}

Each stage of embryos and mature oocyte without zona pellucida were fixed in $4 \%$ paraformaldehyde for $30 \mathrm{~min}$ at $4{ }^{\circ} \mathrm{C}$. Fixed samples were permeabilized by $1 \%$ Triton X-100 for $5 \mathrm{~min}$ at room temperature and washed three times with PBS. The embryos and oocytes were blocked using 1\% BSA in PBS for $1 \mathrm{~h}$ at room temperature and primary antibodies targeting NTDs or CTDs of OCT4 (SC-5279, mouse-anti-OCT4 or SC-8629, goat-anti-OCT4 respectively) were added overnight at $4{ }^{\circ} \mathrm{C}$. Each primary antibody was diluted $1: 100$ in PBS containing 1\% BSA. Embryos were washed three times with PBS containing $0.1 \%$ Tween-20 before incubation with fluorescent-conjugated secondary antibodies, anti-mouse-lgG (1:500, A11029, Invitrogen), and anti-goat-IgG (1:200,
SC-362284, SantaCruz), which were diluted in a blocking solution, for $1 \mathrm{~h}$ at room temperature. The samples were washed three times with PBS containing $0.1 \%$ Tween-20 and the nucleus was stained using $0.1 \%$ Hoechst 33342 (Molecular Probes, Eugene, OR, USA) for $10 \mathrm{~min}$. After washing three times with PBS, samples were mounted on the slide glass. Stained samples were visualized under an LSM 700 Laser Scanning Microscope (Carl Zeiss, Oberkochen, Germany) and captured images were processed using the ZEN 2012 Light Edition Software (Carl Zeiss).

\section{Statistical analysis}

Statistical analysis was performed using the Graphpad Prism statistical program (Graphpad Software, San Diego, CA, USA). Porcine OCT4 variant expression in oocytes and developing preimplantation embryos were examined using one-way ANOVA and Tukey's test. Differential gene expressions between the two classified groups of individual blastocyst were compared using unpaired Student's t-test. Correlation between OCT4 variant and pluripotent gene expression in a single blastocyst was analyzed by the Pearson's correlation coefficient. All data were expressed as mean \pm s.E.M. and $P<0.05$ was considered to be significant.

\section{Results \\ Identification of novel porcine OCT4 variants, OCT4B and OCT4B1}

To examine the presence of porcine OCT4 novel variants, the human OCT4 gene sequence was compared with the porcine genome using BLAST. The results indicated that the $3^{\prime}$ region of the first intron of porcine OCT4 is similar to the first exon of the human $O C T 4 B$ transcript (Fig. 1). Following the alignment, primers were prepared to analyze expression of OCT4 variants in pig (Fig. 2A and Supplementary Table 1). As it has been reported that $O C T 4 B$ is expressed in the human preimplantation embryo (Cauffman et al. 2006), the cDNA samples from IVF blastocysts $(n=15)$ were subjected to RT-PCR. The region from BF3 to R3 was successfully amplified (Fig. 2B) and the PCR product was validated by sequencing (Supplementary Figure 2, see section on supplementary data given at the end of this article). This finding indicated that porcine OCT4B variants also share the second and last exons of porcine OCT4A, as has been observed in human and mice. The additional amplicon, which is $\sim 300$-bp larger than the expected PCR product, was consistently detected when the reverse primer amplifying downstream of the second intron (R2 and R3) was subjected to PCR (Fig. 2B). The sequence of the amplicon demonstrated that the PCR product spans the second intron of porcine OCT4A (Supplementary Figure 2) and that the structure of the variant is similar to that of human OCT4B1 mRNA. Therefore, in this study, we named the variant harboring the second intron in its transcript as porcine OCT4B1. 


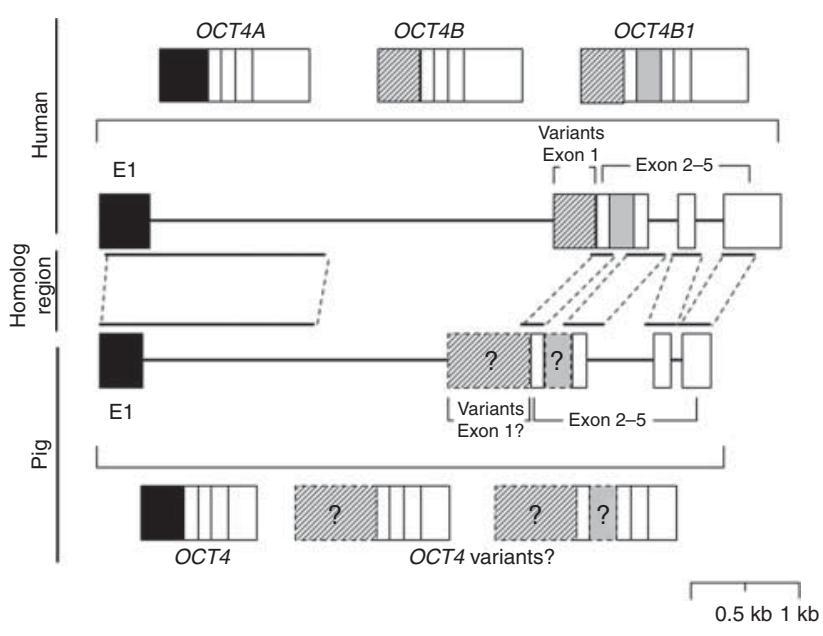

Figure 1 Identification of candidate coding regions of porcine OCT4 variants by searching for regions homologous to human OCT4. The structure and sequence of homologous sites between human and porcine OCT4 are represented. Human OCT4 variants showed differences in the three regions (black, slashed, and gray rectangles). The region homologous to the coding region of human OCT4 variants in the porcine genome was searched by BLAST (black lines). Based on the sequence similarity and location of the first exon of human OCT $4 B$ (slashed box) and OCT4B1-specific exon (gray box), porcine OCT4B and OCT4B1-specific sites were determined (boxes with question marks). The diagram is scaled.

Based on the identified partial sequence of novel porcine OCT4 variants, RACE PCR was carried out to confirm the full sequence of each variant. Both variants share the TSSs, which is located 432 bp upstream from the second exon of porcine OCT4A (Fig. 2C). The last sequence of both variants was identical to that of the porcine OCT4A transcript (Fig. 2D). Identified new porcine OCT4 variants, OCT4B and OCT4B1 (GenBank accession nos KJ023671 and KJ023672 respectively), have distinct first exons (E1B) compared with the $A$ form, and OCT4B1 has a cryptic exon (E2B), which is in the intron region in porcine OCT $4 A$ and $O C T 4 B$, as it is in human OCT4B1 (Fig. 2E). These results revealed that porcine OCT4 transcribes additional mRNAs, OCT $4 B$ and OCT4B1, as well as OCT4A.

\section{Designing an OCT4A-specific primer pair}

Before analyzing expression of OCT4 variant in various tissues and embryonic stage, OCT4A-specific primer pairs were designed first because the OCT4A pseudogenes would complicate the attempts made to measure accurate amounts of OCT4A mRNA (Liedtke et al. 2007). As human OCT4 pseudogenes do not have introns, and two genomic sequences similar to the porcine OCT4Aspecific exon, E1A, and E2, were searched in this study (Supplementary Figure 3, see section on supplementary data given at the end of this article), gDNA PCR was carried out to investigate whether or not the designed primer pairs amplify porcine OCT4A pseudogenes.
Production of amplicon with the expected size resulted from gDNA PCR means that the primer pair detects pseudogene, and concluded that the pair is not desirable for distinguishing between porcine OCT4A and its pseudogenes. Examined primer sets did not target the pseudogene except AF1-AR1 pair, and the AF2-AR2 pair was determined to be the most desirable primer pair because it detected the fewest number of non-specific amplicons among them (Supplementary Figure 4A). The AF2-AR2 primer pair was used to examine OCT4A expression in various porcine tissues and successful amplification was detected only in testis (Fig. 3A) without non-specific products detected from the gDNA amplification. The amplicon was sequenced and no OCT4 pseudogenes were detected (Supplementary Figure 4B). Therefore, the designed primer pair in this study is suitable for specifically detecting porcine OCT $4 A$ mRNA that allows the accurate quantification of OCT4A mRNA with a reproducible and reliable discrimination among porcine OCT4 variants.

\section{Expression of OCT4 variants in adult tissue}

The expression of porcine OCT4 variants was examined in six male somatic tissues - liver, lung, kidney, heart, spleen, and testis. Porcine OCT $4 B$ was expressed in all tissues but OCT4B1 was detected only in testis (Fig. 3A). The PCR products were sequenced and the amplicon was confirmed as the targeted product. After examining mRNA expression of each variant in tissues, the open reading frames (ORFs) from OCT $4 B$ and OCT $4 B 1$ transcripts were analyzed (Supplementary Table 5, see section on supplementary data given at the end of this article) using the RevTrans ORF finder (Wernersson \& Pedersen 2003). Three and eight ORFs starting with the ATG and CTG start codons, respectively, were identified from the OCT $4 B$ template sequence (Supplementary Table 5). The ORF from OCT $4 B 1$ produced a truncated protein because of the stop codon in the E2B, as has been observed in human OCT4B1. As an antibody targeting OCT4B1 was not available commercially, only OCT4B expression was examined by western blot in this study. The protein detected by OCT4 C-terminal targeting antibody (SC-8629) was slightly $<25 \mathrm{kDa}$ (Fig. 3B). Compared with the identified ORF information, the detected protein would be OCT4B-201AA (22.38 kDa). However, OCT4A was not detected in testis tissues even though we used various OCT4A-specific antibodies as well as CTDs targeting OCT4 antibody (data not shown). This absence could be a result of a heterogenic cell population in assessed testis tissue and a low proportion of OCT4A-positive cells. The results indicate that porcine $O C T 4 B$ has protein-coding variants, as has been observed in other species, and it is broadly expressed in adult tissues, in contrast to OCT $4 A$ and OCT $4 B 1$ of which mRNAs were expressed only in testis. 


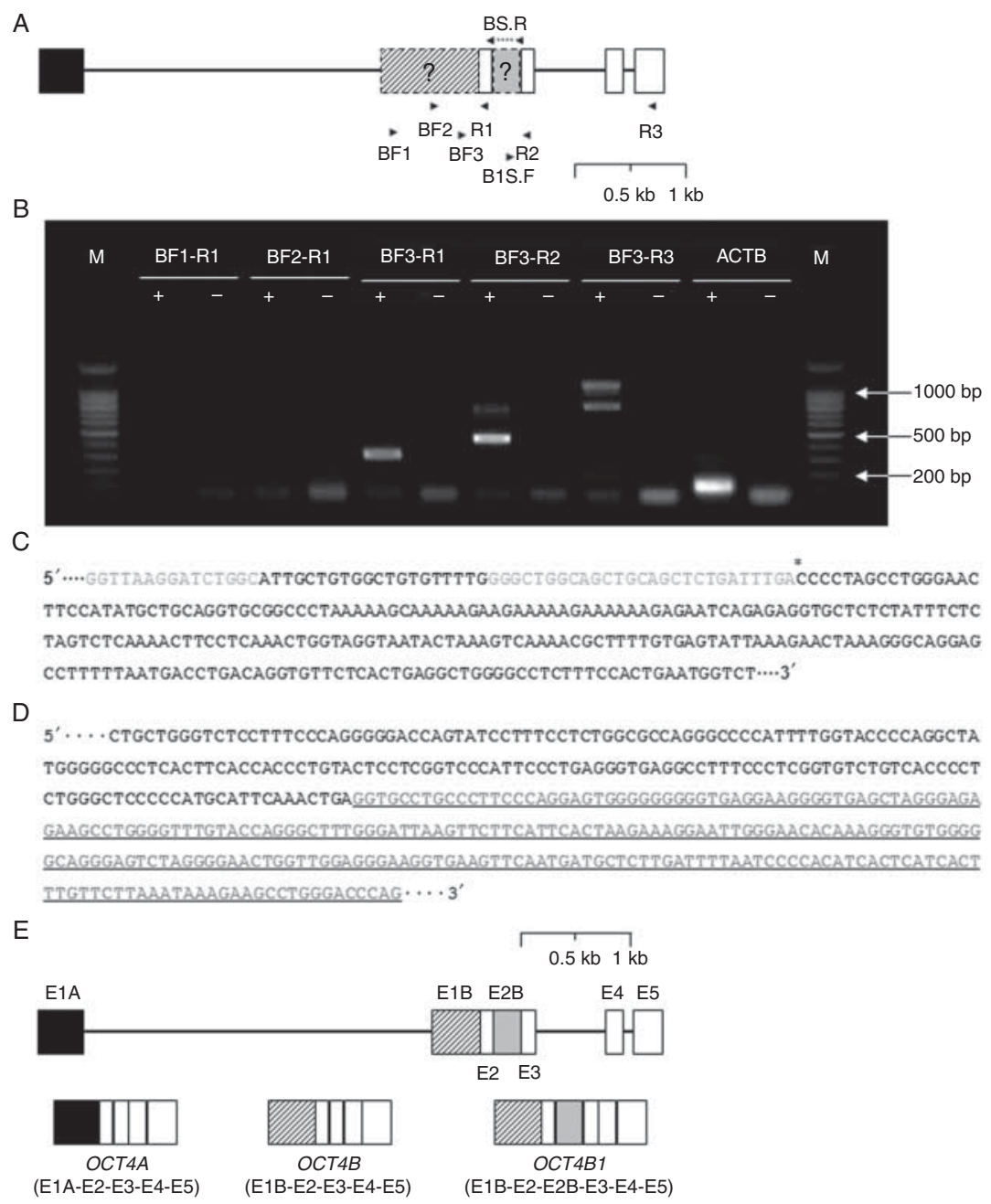

Figure 2 Identification of porcine OCT4 variants, OCT $4 B$ and OCT4B1. (A) Porcine OCT4 gene structure and anticipated variants. Rectangles indicate exon 1 (black) and exons 2-5 (white). Boxes with question marks indicate possible coding regions for OCT $4 B$ and OCT $4 B 1$ transcripts. Primers are shown and the primer noted as BS.R indicates the primer located between exons 2 and 3 used to distinguish OCT $4 B$ and OCT $4 B 1$. The diagram is scaled. (B) Candidate OCT4 variant expression in porcine blastocyst. The cDNA $(+)$ from pooled blastocyst $(n=15)$ was successfully used to amplify the BF3 to R3 region. Non-reverse transcribed RNA (-) was used for a negative control and $\mathrm{M}$ indicates the $100 \mathrm{bp}-\mathrm{DNA}$ ladder. (C) Identified transcription starting sites (TSSs). The sequence marked by an asterisk is the first sequence of the novel OCT4 variant. Characters in boxes indicate BF2 (upper) and BF3 (lower) primers. Black sequences and gray sequences represent expression and non-expression sites respectively. (D) Identified last sequences. Last sequences were the same as those previously reported for the OCT $4 A$ mRNA sequence. Text in box indicates the R3 primer complimentary sequences. Black characters and gray characters with underline represent exon 5 and the $3^{\prime}$-UTR respectively. (E) Identified porcine OCT4 gene structure and variant mRNA. Boxes and lines indicate exons and introns respectively. Black, slash, and gray boxes are OCT $4 A-$, OCT $4 B-$, and $O C T 4 B 1$-specific regions respectively. The diagram is scaled.

\section{Comparative analysis of mRNA of OCT4 variants in porcine oocytes and preimplantation embryos}

After identification of the novel porcine OCT4 variants, $O C T 4 B$ and OCT4B1, we examined the expression quantity of each variant in mature oocytes and developing preimplantation embryos (Fig. 4). All variants were expressed in oocytes and developing embryos. The amounts of OCT $4 A$ and $O C T 4 B$ transcripts per embryo and oocyte were significantly higher in the blastocyst stage compared with other embryonic stages (Fig. 4A). After analyzing the expression of each variant in oocytes and developing embryos, the proportions of variants in each embryonic stage were examined (Fig. 4B). The OCT $4 B$ and OCT $4 B 1$ mRNA comprised $\sim 7-19$ and $<1-7 \%$, respectively, among porcine OCT4 variants in the developing embryo. The proportion of OCT4A transcript was significantly higher compared with that of other variants in all examined preimplantation embryonic stages. This finding indicates that the OCT $4 A$ transcript is dominantly expressed in porcine embryonic stage rather than newly identified OCT $4 B$ and OCT $4 B 1$ mRNAs.

\section{Porcine OCT4A and OCT4B expression and location in oocytes and preimplantation embryos}

The expression and locations of OCT4A and OCT4B were examined in mature oocytes and developing embryos (Fig. 5). Owing to the lack of antibody to OCT4B1, it was excluded from these experiments. OCT4A was detected in the blastocyst (Fig. 5A) but OCT4B was detected in all embryonic stages and mature oocytes (Fig. 5B). Interestingly, each protein had distinct locations in the embryo. OCT4A was detected only in the nucleus, while expression of OCT4B was found in the cytoplasm and membrane. However, the antibody targeting OCT4 CTD, which was expected to detect both the $A$ and $B$ forms of OCT4, showed unclear specificity for detection of OCT4A because the certain expression in nucleus was not observed in the blastocyst (dashed area in Fig. 5B), which showed positive expression of OCT4A (Fig. 5A). These results indicate that the OCT4B protein is expressed in differential stages of and locations in the embryo compared with OCT4A expression. 


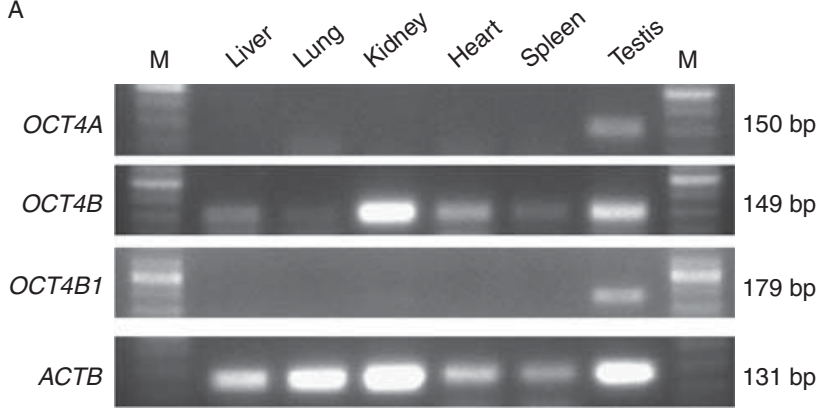

B

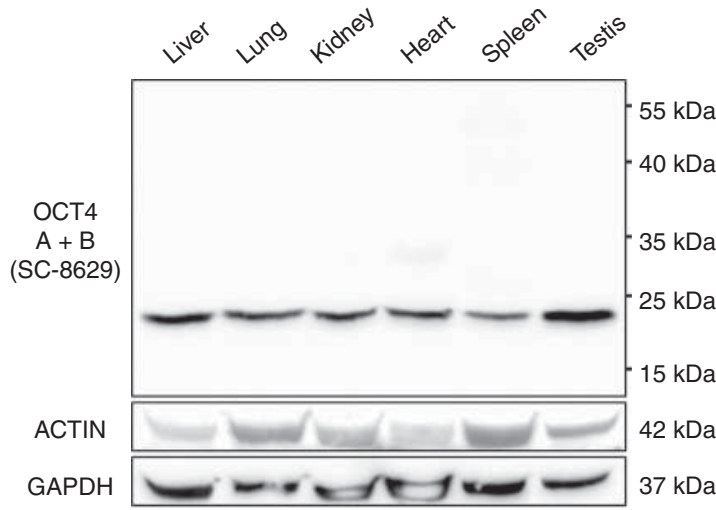

Figure 3 Expression of porcine OCT4 variants in somatic tissue. (A) Discriminative analysis of porcine OCT4 variant transcript expression in adult tissue. Expression of each variant in adult tissues was examined discriminatively. $M$ indicates the 100 bp DNA ladder. Each PCR product was confirmed by sequencing. (B) OCT4B expression in somatic tissue. Fifty micrograms of whole protein from each tissue were assessed by western blot. The antibody that detects the C-terminal OCT4 domain was used to examine the B form of OCT4 production in porcine tissues. ACTIN and GAPDH were used as internal controls.

\section{Correlation of OCT4 variants with other pluripotency-associated gene expression}

One research group has suggested that OCT4B1 could be used as a pluripotent marker in human (Atlasi et al. 2008), in contrast to OCT4B, which is considered to be less related to pluripotency (Lee et al. 2006a, Guo et al. 2012). Therefore, in order to clarify as to what relationship exists between the identified variants and pluripotency in pig, the expression levels of the variants were compared with pluripotent genes (OCT4A, SOX2, and $N A N O G$ ) and the trophectodermal marker gene (CDX2) in the blastocyst (day $=7, n=40$ ). The blastocysts were classified into two groups expressing over or less than the median amount of OCT $4 B$ and OCT4B1, and the expression levels of OCT4A, SOX2, NANOG, and $C D X 2$ in both groups were compared with each other (Fig. 6). The expression levels of the four examined genes were not significantly different in the two groups divided by the OCT $4 B$ mRNA expression level $(P>0.05$, Fig. 6A). However, the groups classified by OCT4B1 expression showed significantly different levels of expression for SOX2, NANOG, and CDX2 $(P<0.05$, Fig. 6B). After comparison of the gene expression levels between the two groups, we analyzed the correlation between each variant and OCT4A, SOX2, NANOG, and $C D X 2$. The expression of OCT4B1 was negatively correlated with SOX2 and NANOG expression, but positively for $C D X 2(P<0.05$, Fig. 7). Unexpectedly, $O C T 4 B$ expression was positively correlated with $C D X 2$ $(P<0.05$, Supplementary Figure 6 , see section on supplementary data given at the end of this article), even though the expression levels between OCT4Bhigh and -low blastocysts were not significantly different (Fig. 6A).
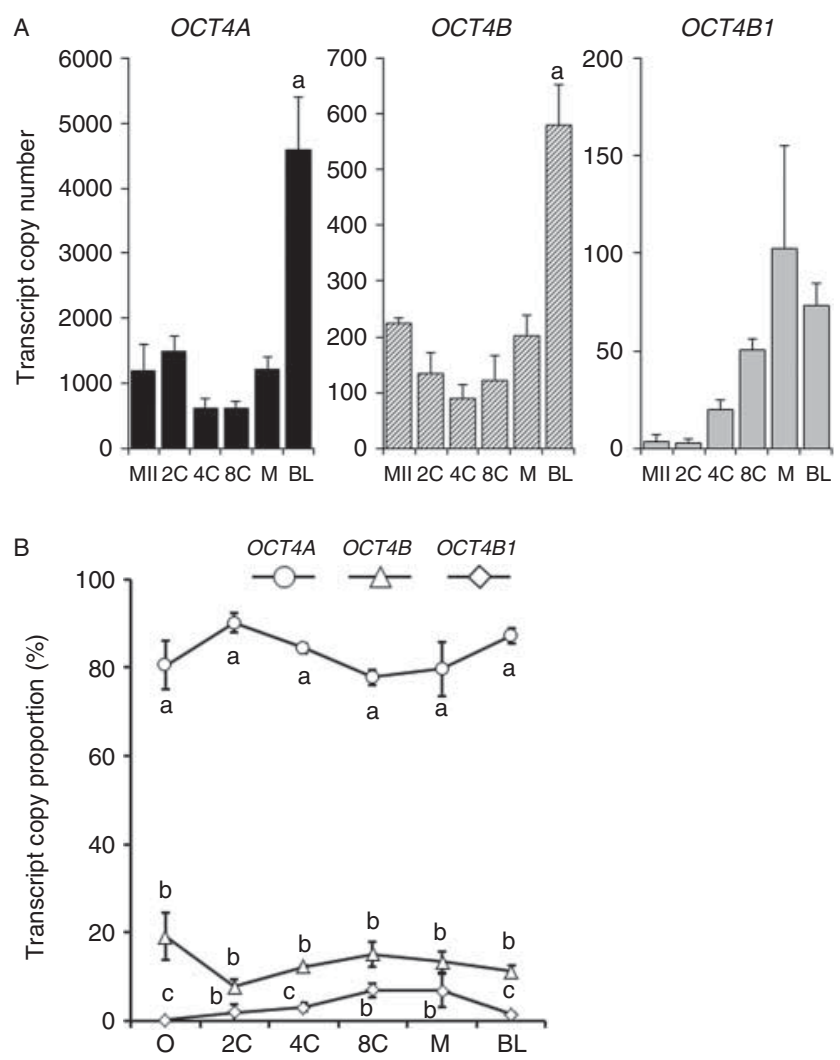

Figure 4 Quantitative comparison of mRNA of each porcine OCT4 variant in mature oocytes and developing preimplantation embryos. (A) The mRNA copy number of each variant in the developing embryo. The absolute amount of each variant was examined discriminatively in mature oocyte (MII, $n=40$ ) and 2-cell (2C, $n=20)$, 4-cell (4C, $n=20$ ), 8-cell (8C, $n=20)$, morula (M, $n=10)$, and blastocyst (BL, $n=5)$ preimplantation embryonic stages. The copy number of each variant in an oocyte and embryo was calculated using an equation described in the 'Materials and methods' section. The data are expressed as mean \pm S.E.M. Different characters indicate significant differences $(P<0.05)$. (B) Each variant proportion in oocytes and developing embryos. The proportion of each variant was analyzed by comparing the copy numbers of each variant in oocytes $(\mathrm{O})$ and preimplantation embryos (2-cell, 2C; 4-cell, 4C; 8-cell, 8C; morula, M; and blastocyst, $\mathrm{BL})$. The data are expressed as mean \pm s.E.M. Different characters indicate significant differences $(P<0.05)$. 


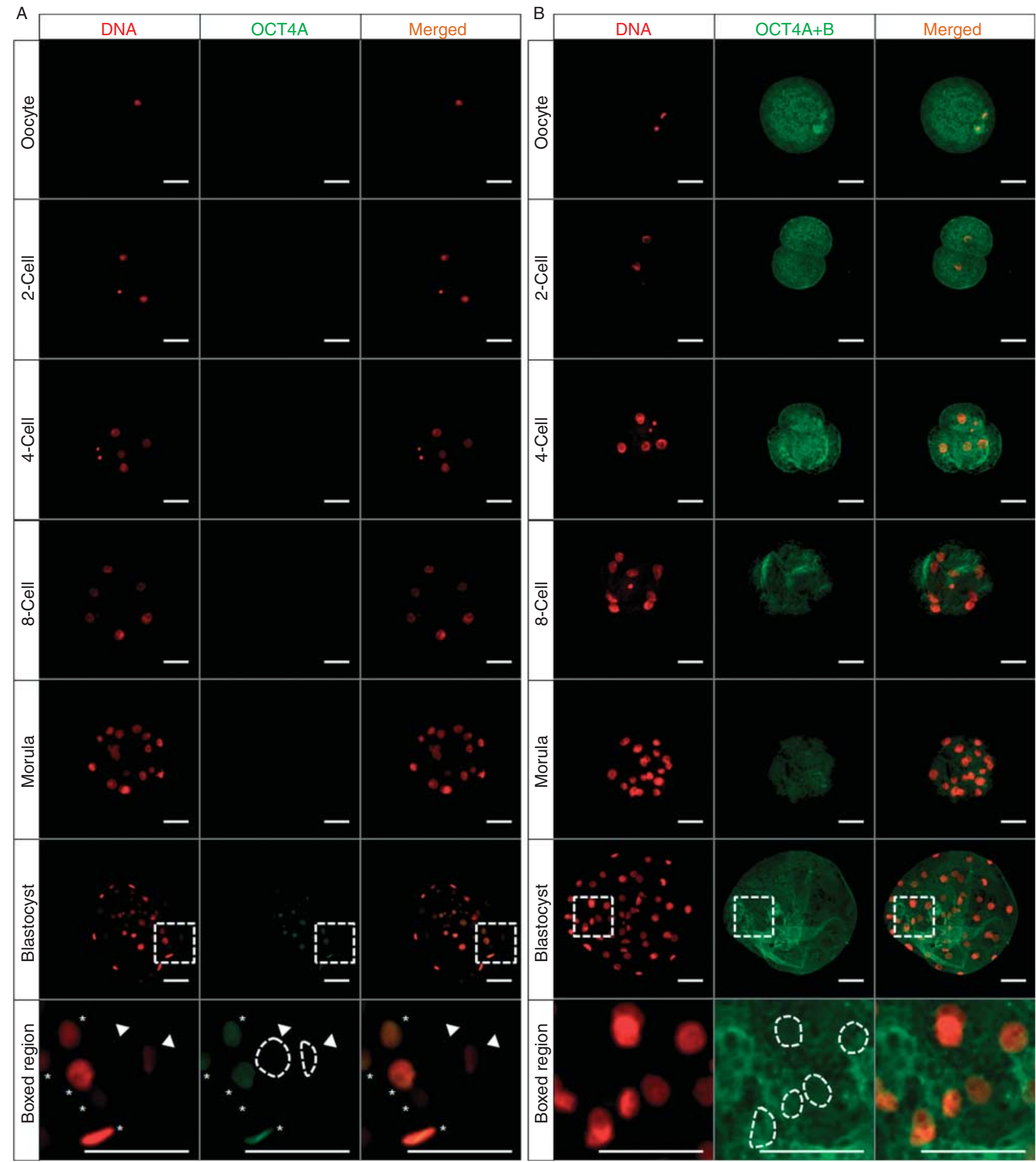

Figure 5 OCT4 variant protein expression in oocytes and preimplantation embryos. OCT4A (A) and, both OCT4A and OCT4B (B) protein expression was examined in oocytes and developing embryos. DNA and protein targeted by each antibody are represented in red and green colors respectively. Asterisks and arrowheads indicate OCT4A-positive and -negative cells in blastocyst respectively. The area with a dashed line in the magnified region shows the DNA-stained area. Staining with secondary antibodies without primary antibodies was used as a negative control (Supplementary Figure 5). Scale bar $=50 \mu \mathrm{m}$.

\section{Discussion}

Previous studies have suggested that Oct4, especially Oct4A, is the most important gene for maintaining pluripotency in ES cells, acquisition of pluripotency in somatic cells, and normal development in the preimplantation embryo (Nichols et al. 1998, Niwa et al. 2000, Takahashi \& Yamanaka 2006). These studies 


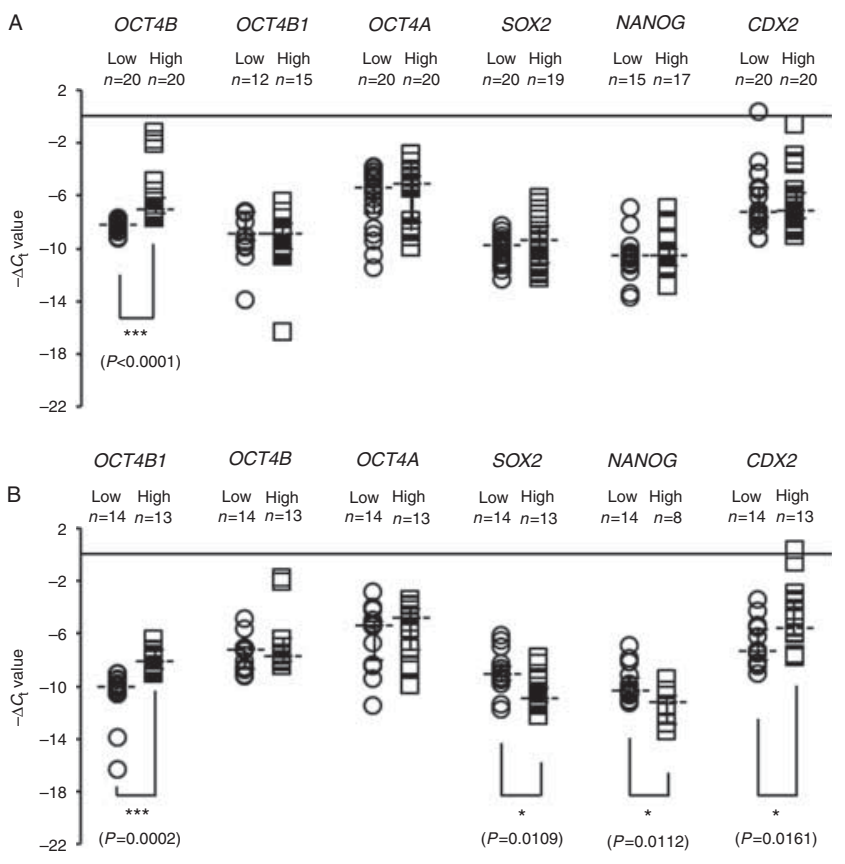

Figure 6 Comparison of pluripotent gene expression between two groups of individual blastocyst classified by the expression level of each variant. The aligned dot plot shows the relative quantity of the examined genes from a single blastocyst classified by expression abundance of OCT4B (A) and OCT4B1 (B). The median of OCT4B $(n=40)$ and OCT4B1 $(n=27)-\Delta C$ t values $(-7.7908$ and -9.1157 respectively) were used as standards for grouping individual blastocysts. $-\Delta C$ t values of the genes in the blastocyst variant-high group and variant-low group are indicated by circles and squares respectively. The box plot in each column indicates quartiles. $A C T B$ was used as a reference gene and asterisks show significant differences between two groups $\left({ }^{*} P<0.05\right.$ and $* * * P<0.001)$.

have made OCT4A an essential gene for analyzing stem cell status and embryo development in various species, including pig. However, compared with the OCT4A, other variants have been less focused even though several years have passed since the identification of the variant in human and mice (Takeda et al. 1992, Mizuno \& Kosaka 2008). The OCT4 variants have been focused with regard to the following two aspects generally: i) distinct properties compared with OCT4A (Cauffman et al. 2006, Lee et al. 2006a, Atlasi et al. 2008, Guo et al. 2012) and ii) an inducer making the improper OCT4A detection (Wang \& Dai 2010). However, these studies have also been restricted to human and mice until recently, and even the presence of OCT4 variants is still unclear in pig. Therefore, careful insights into the OCT4 variant are required for the comprehensive understanding of the OCT4 gene as well as accurate analysis of OCT4A in pig. Therefore, undefined porcine OCT4 variants, OCT $4 B$ and $O C T 4 B 1$, have been confirmed and the expression of each variant was examined independently in developing porcine embryos in this study.

\section{The gene structure of porcine $\mathrm{OCT} 4 \mathrm{~B}$ and OCT4B1 are conserved}

OCT4A sequences and domains among species were highly conserved and the function of the gene has been suggested to be an ancient trait of vertebrates (Tapia et al. 2012). The structure of variant mRNA is similar between human and mice, including POU- and CTD-coding exons with differential NTD-coding exon compared with the A form. However, the sequence of the OCT4Bspecific exon and NTDs were not conserved between human and mice (Mizuno \& Kosaka 2008, Guo et al. 2012). According to our result, the porcine genome aligned to the human OCT $4 B$-specific exon with low homology (Fig. 1), but the transcript structure was similar to that of the previously reported $B$ form of OCT4 in human (Fig. 2). The other OCT4 variant, OCT4B1, was identified only in human (Atlasi et al. 2008). This variant has a cryptic exon (the second intron in OCT4) and a stop codon is present on the exon (E2B). Although the lengths and sequences of the OCT4B1-specific exons (E2B) were not similar between human and pig (Fig. 1), the variant structure is similar to that of human OCT $4 B 1$ (Fig. 2) and there is a stop codon in the porcine OCT4B1specific exon, as in human (Supplementary Table 5). These mean that the transcript structure of porcine $O C T 4 B$ and OCT4B1, not their sequences, are conserved in those of mice and human.

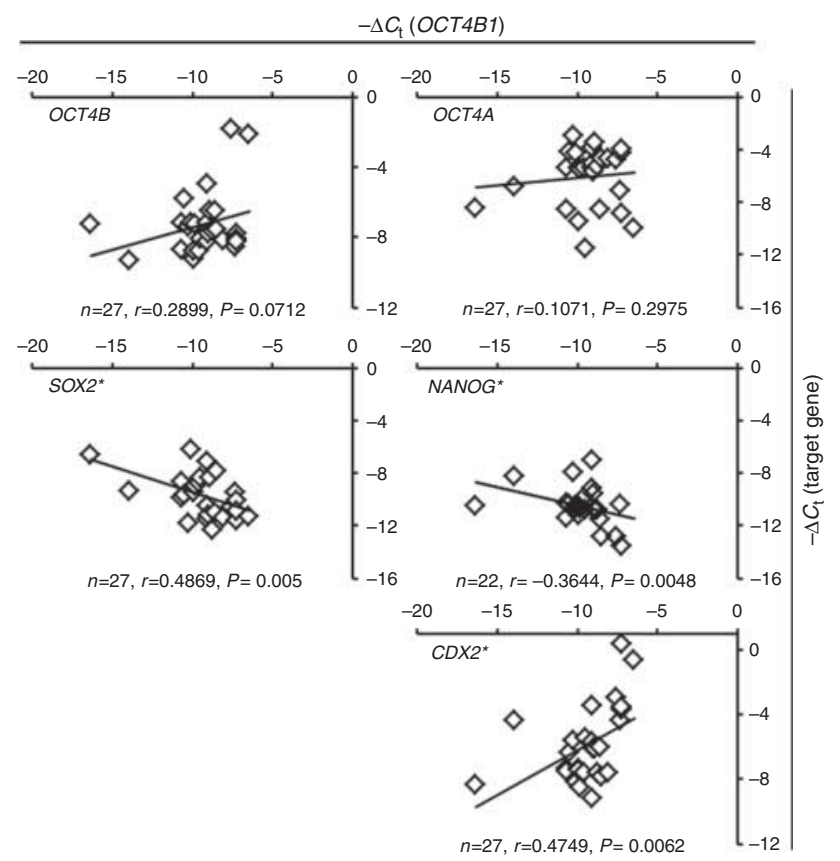

Figure 7 Correlative expression analysis of OCT4B1 and pluripotent genes in individual blastocyst. The correlative expression between OCT $4 B 1$ and other variants (OCT4A, $n=27$ and OCT $4 B, n=27)$ or pluripotent genes (SOX2, $n=27$; NANOG, $n=22$; and $C D X 2, n=27$ ) was examined. $P<0.05$ is considered to indicate a significant correlation by the Pearson's correlation coefficient. $X$-axis and $y$-axis indicate the $-\Delta C$ t value of OCT $4 B 1$ and examined genes respectively. 


\section{Porcine OCT4B $m R N A$ generate protein}

Transcribed OCT $4 B$ mRNA has been shown to produce three alternatively translated proteins in mice and human (Wang et al. 2009, Guo et al. 2012). These reports also suggested that a few proteins, which are smaller than the protein produced from the first translation-starting site, were produced by an internal ribosomal entry site (IRES). Although an $\sim 25 \mathrm{kDa}$-sized protein, which would be translated by the IRES mechanism as in other species, was detected only in our study (Fig. 3B), it is hard to eliminate the possibility that the other sizes of proteins could have been produced alternatively in other tissues, which were not examined in this study. Therefore, it is still necessary to examine the presence of alternatively translated proteins by transfecting the OCT4B construct in pig cells. In addition, the starting codon of the detected protein is unclear because the possible porcine OCT4B ORFs are different compared with mouse and human B-form ORFs. Hence, confirmation of the exact starting codon is also needed. Meanwhile, our results indicate that the porcine $O C T 4 B$ transcript produces a novel protein, which is different from what has been previously reported for OCT4A. The OCT4B1 mRNA was found to be generated as a truncated protein, owing to the presence of a stop codon (TGA) in the cryptic exon (Farashahi Yazd et al. 2011). Although pig is expected to also produce truncated OCT4B1, as in human, whether or not it does is unclear because of the lack of a proper antibody. Therefore, the presence of porcine OCT4B1 needs to be studied further.

\section{All of OCT4 variants are expressed in developing embryo}

Quantitative analyses of OCT4 expression in porcine embryonic stage have been performed in a few previous studies (Lee et al. 2006b, Magnani \& Cabot 2008), but the expression patterns of the variants were not examined in pig. It was confirmed that the expression patterns of $O C T 4 B$ were similar to that of OCT4A, but OCT4B1 expression was variable (Fig. 4A). The proportion of each variant in embryonic stage showed that the variants occupied small proportion (Fig. 4B) as in human ES cells (Lee et al. 2006a). This means that OCT4A is mainly expressed in developing porcine embryos compared with other two variants, OCT $4 B$ and OCT $4 B 1$ forms. The protein produced from each variant was also expressed in different stages and located distinctly in the developing embryo (Fig. 5). In human, OCT4B was produced in the earlier embryonic stages with a different location compared with expression of OCT4A (Cauffman et al. 2006). As in human, although the exact embryonic stage expressing each variant was different in pig, the results were similar in that OCT4B was present in the embryo cytoplasm and was expressed in different embryonic stages compared with what has been observed for OCT4A. One report showed that the NTD of OCT4A is essential for incorporation of the protein into the nucleus (Lee et al. 2006a). Porcine OCT4B ORFs showed the deletion of the OCT4A NTD (Supplementary Table 5). This deletion could result in the differential location of OCT4B compared with OCT4A in pig. These expression patterns of OCT4 variants in embryonic stage demonstrate that they would have the functions in embryo, but the role of the variants could be distinct from each other in pig.

\section{OCT4B would not be a 'pluripotent' marker in pig}

The roles of $O C T 4$ novel variants are still unclear, in contrast to that of their major form, OCT4A. OCT4B expression was confirmed in human pancreatic islet (Takeda et al. 1992) and mouse eye, especially in retinal pigment epithelial cells (Mizuno \& Kosaka 2008). The expression of OCT4B in somatic tissue indicates that the variant could have a different function compared with OCT4A and would have little relationship with pluripotency. In human ES cells, $O C T 4 B$ could not maintain pluripotency in place of OCT4A and it failed to bind to the octamer consensus despite the presence of the POU domain (Lee et al. 2006a). Furthermore, one report demonstrated that Oct $4 B$ failed to reprogram somatic cells into pluripotent stem cells when co-infected with other defined reprogramming factors, Sox2, Myc, and Klf4 in mice (Guo etal. 2012). These studies clearly demonstrated that $O C T 4 B$ is not related to pluripotency. In our study, OCT $4 B$ was detected in all examined somatic tissues (Fig. 3A), and cytoplasmic localization of OCT4B (Fig. 5B) showed that the protein would not be a transcription factor. The non-significant correlation with pluripotent gene expression in individual blastocyst (Fig. 6A and Supplementary Figure 6) also supports the notion that $O C T 4 B$ would not be a pluripotent marker in pig. Although the correlative study suggests restricted information compared with the biological approach, these results demonstrated the possibility that $O C T 4 B$ could be involved in biological functions other than pluripotency in pig, as in other species. Some reports demonstrated that $O C T 4 B$ expression was affected by heat and oxidative stress (Wang et al. 2009, Guo et al. 2012). Therefore, it is possible that porcine OCT $4 B$ could have a function related to stress response. These previous reports and expression patterns in tissues and preimplantation embryos indicate that OCT $4 B$ would not be related to pluripotency as OCT4A.

\section{Is $\mathrm{OCT} 4 \mathrm{~B} 1$ related to pluripotency in pig?}

The other OCT4 variant, OCT4B1, which has been previously identified only in human (Atlasi et al. 2008), has been suggested to be involved in pluripotency. Atlasi et al. identified the OCT4B1 transcript and suggested that $O C T 4 B 1$ could have a pluripotency-related function for the following three reasons: i) OCT4B1 was highly 
expressed in ES and EC cells compared with somatic cells; ii) differentiated ES cells lose OCT4B1 expression; and iii) the SSEA3-positive cell population in Shef5 human ES cells strongly expressed OCT4B1. Although these results could indicate that OCT $4 B 1$ might be an alternative pluripotent marker (Papamichos et al. 2009), the exact biological function of OCT4B1 is still unclear. In our study, porcine OCT4B1 was detected in embryonic stage and testis only among the examined tissues (Figs 2 and 3A), and correlative expression between OCT4B1 and pluripotent genes was observed (Fig. 7). Although these results would mean that OCT4B1 might be related to the pluripotency in pigs, it is hard to confirm whether the variant is associated with pluripotency as the results in this study provide only limited evidence via indirect analysis. Some studies tried to confirm the biological function of OCT4B1. Knockdown of OCT4B1 increased caspase- 3 and -7 activity and induced apoptosis in gastric cancer (Asadi et al. 2011), and heat stress increased OCT4B1 expression (Farashahi Yazd et al. 2011). Therefore, proper understanding of the exact function of OCT4B1 and its relationship with pluripotency needs further studies.

In this study, porcine OCT4 variants were identified first. There is a report addressing that discriminative analysis of OCT4 variants is required because of their similarity in structure (Wang \& Dai 2010). As in other species, the presence of porcine OCT4 variants suggests that OCT4 gene is analyzed carefully by distinguishing other variants also in pigs. Examination of porcine OCT4 variant expression was carried out in adult tissue, mature oocytes, and developing embryos, and it was confirmed that each variant has differential expression patterns. For the comprehensive understanding of porcine OCT4 genes, OCT4A as well as OCT $4 B$ and OCT $4 B 1$, their functional studies should be conducted further.

\section{Supplementary data}

This is linked to the online version of the paper at http://dx.doi. org/10.1530/REP-14-0403.

\section{Declaration of interest}

The authors declare that there is no conflict of interest that could be perceived as prejudicing the impartiality of the research reported.

\section{Funding}

This work was supported by Basic Science Research Program through the National Research Foundation of Korea (NRF) funded by the Ministry of Education (NRF-2014055199) and Next BioGreen 21 program, Rural Development Administration, Republic of Korea.

\section{References}

Abeydeera LR \& Day BN 1997 In vitro penetration of pig oocytes in a modified Tris-buffered medium: effect of BSA, caffeine and calcium. Theriogenology 48 537-544. (doi:10.1016/S0093-691X(97)00270-7)

Asadi MH, Mowla SJ, Fathi F, Aleyasin A, Asadzadeh J \& Atlasi Y 2011 OCT4B1, a novel spliced variant of OCT4, is highly expressed in gastric cancer and acts as an antiapoptotic factor. International Journal of Cancer 128 2645-2652. (doi:10.1002/ijc.25643)

Atlasi Y, Mowla SJ, Ziaee SA, Gokhale PJ \& Andrews PW 2008 OCT4 spliced variants are differentially expressed in human pluripotent and nonpluripotent cells. Stem Cells 26 3068-3074. (doi:10.1634/stemcells. 2008-0530)

Cauffman G, Liebaers I, Van Steirteghem A \& Van de Velde H 2006 POU5F1 isoforms show different expression patterns in human embryonic stem cells and preimplantation embryos. Stem Cells $\mathbf{2 4}$ 2685-2691. (doi:10.1634/stemcells.2005-0611)

Farashahi Yazd E, Rafiee MR, Soleimani M, Tavallaei M, Salmani MK \& Mowla SJ 2011 OCT4B1, a novel spliced variant of OCT4, generates a stable truncated protein with a potential role in stress response. Cancer Letters 309 170-175. (doi:10.1016/j.canlet.2011.05.027)

Funahashi H, Cantley TC \& Day BN 1997 Synchronization of meiosis in porcine oocytes by exposure to dibutyryl cyclic adenosine monophosphate improves developmental competence following in vitro fertilization. Biology of Reproduction 57 49-53. (doi:10.1095/biolreprod57.1.49)

Guo CL, Liu L, Jia YD, Zhao XY, Zhou Q \& Wang L 2012 A novel variant of Oct3/4 gene in mouse embryonic stem cells. Stem Cell Research $\mathbf{9}$ 69-76. (doi:10.1016/j.scr.2012.04.004)

Hwang JY, Kim EB, Ka H \& Lee CK 2013a Identification of the porcine XIST gene and its differential CpG methylation status in male and female pig cells. PLoS ONE 8 e73677. (doi:10.1371/journal.pone.0073677)

Hwang JY, Mulligan BP, Kim HM, Yang BC \& Lee CK $2013 b$ Quantitative analysis of sperm mRNA in the pig: relationship with early embryo development and capacitation. Reproduction, Fertility, and Development 25 807-817. (doi:10.1071/RD12160)

Lee J, Kim HK, Rho JY, Han YM \& Kim J 2006a The human OCT-4 isoforms differ in their ability to confer self-renewal. Journal of Biological Chemistry 281 33554-33565. (doi:10.1074/jbc.M603937200)

Lee E, Lee SH, Kim S, Jeong YW, Kim JH, Koo OJ, Park SM, Hashem MA, Hossein MS, Son HY et al. 2006b Analysis of nuclear reprogramming in cloned miniature pig embryos by expression of Oct- 4 and Oct- 4 related genes. Biochemical and Biophysical Research Communications 348 1419-1428. (doi:10.1016/j.bbrc.2006.08.004)

Liedtke S, Enczmann J, Waclawczyk S, Wernet P \& Kogler G 2007 Oct4 and its pseudogenes confuse stem cell research. Cell Stem Cell $\mathbf{1}$ 364-366. (doi:10.1016/j.stem.2007.09.003)

Magnani L \& Cabot RA 2008 In vitro and in vivo derived porcine embryos possess similar, but not identical, patterns of Oct4, Nanog, and Sox 2 mRNA expression during cleavage development. Molecular Reproduction and Development 75 1726-1735. (doi:10.1002/mrd.20915)

Mirzaei MR, Najafi A, Arababadi MK, Asadi MH \& Mowla SJ 2014 Altered expression of apoptotic genes in response to OCT4B1 suppression in human tumor cell lines. Tumour Biology 35 9999-10009. (doi:10.1007/ s13277-014-2238-9)

Mizuno N \& Kosaka M 2008 Novel variants of Oct-3/4 gene expressed in mouse somatic cells. Journal of Biological Chemistry 283 30997-31004. (doi:10.1074/jbc.M802992200)

Nichols J, Zevnik B, Anastassiadis K, Niwa H, Klewe-Nebenius D, Chambers I, Scholer H \& Smith A 1998 Formation of pluripotent stem cells in the mammalian embryo depends on the POU transcription factor Oct4. Cell 95 379-391. (doi:10.1016/S0092-8674(00)81769-9)

Niwa H, Miyazaki J \& Smith AG 2000 Quantitative expression of Oct-3/4 defines differentiation, dedifferentiation or self-renewal of ES cells. Nature Genetics 24 372-376. (doi:10.1038/74199)

Okamoto K, Okazawa H, Okuda A, Sakai M, Muramatsu M \& Hamada H 1990 A novel octamer binding transcription factor is differentially expressed in mouse embryonic cells. Cell 60 461-472. (doi:10.1016/ 0092-8674(90)90597-8)

Papamichos SI, Kotoula V, Tarlatzis BC, Agorastos T, Papazisis K \& Lambropoulos AF 2009 OCT4B1 isoform: the novel OCT4 alternative spliced variant as a putative marker of stemness. Molecular Human Reproduction 15 269-270. (doi:10.1093/molehr/gap018) 
Pesce M, Wang X, Wolgemuth DJ \& Scholer H 1998 Differential expression of the Oct-4 transcription factor during mouse germ cell differentiation. Mechanisms of Development 71 89-98. (doi:10.1016/S0925-4773(98) 00002-1)

Rosner MH, Vigano MA, Ozato K, Timmons PM, Poirier F, Rigby PW \& Staudt LM 1990 A POU-domain transcription factor in early stem cells and germ cells of the mammalian embryo. Nature 345 686-692. (doi:10.1038/345686a0)

Scholer HR, Ruppert S, Suzuki N, Chowdhury K \& Gruss P 1990 New type of POU domain in germ line-specific protein Oct-4. Nature $\mathbf{3 4 4}$ 435-439. (doi:10.1038/344435a0)

Takahashi K \& Yamanaka S 2006 Induction of pluripotent stem cells from mouse embryonic and adult fibroblast cultures by defined factors. Cell 126 663-676. (doi:10.1016/j.cell.2006.07.024)

Takahashi K, Tanabe K, Ohnuki M, Narita M, Ichisaka T, Tomoda K \& Yamanaka S 2007 Induction of pluripotent stem cells from adult human fibroblasts by defined factors. Cell 131 861-872. (doi:10.1016/ j.cell.2007.11.019)

Takeda J, Seino S \& Bell GI 1992 Human Oct3 gene family: cDNA sequences, alternative splicing, gene organization, chromosomal location, and expression at low levels in adult tissues. Nucleic Acids Research 20 4613-4620. (doi:10.1093/nar/20.17.4613)
Tapia N, Reinhardt P, Duemmler A, Wu G, Arauzo-Bravo MJ, Esch D, Greber B, Cojocaru V, Rascon CA, Tazaki A et al. 2012 Reprogramming to pluripotency is an ancient trait of vertebrate Oct4 and Pou2 proteins. Nature Communications 3 1279. (doi:10.1038/ncomms2229)

Wang X \& Dai J 2010 Concise review: isoforms of OCT4 contribute to the confusing diversity in stem cell biology. Stem Cells 28 885-893. (doi:10.1002/stem.542)

Wang X, Zhao Y, Xiao Z, Chen B, Wei Z, Wang B, Zhang J, Han J, Gao Y, Li L et al. 2009 Alternative translation of OCT4 by an internal ribosome entry site and its novel function in stress response. Stem Cells 27 1265-1275. (doi:10.1002/stem.58)

Wernersson R \& Pedersen AG 2003 RevTrans: multiple alignment of coding DNA from aligned amino acid sequences. Nucleic Acids Research 31 3537-3539. (doi:10.1093/nar/gkg609)

Yoshioka K, Suzuki C, Tanaka A, Anas IM \& Iwamura S 2002 Birth of piglets derived from porcine zygotes cultured in a chemically defined medium. Biology of Reproduction 66 112-119. (doi:10.1095/biolreprod66.1.112)

Received 7 August 2014

First decision 10 September 2014

Accepted 22 October 2014 